\title{
Double Inflation in Supergravity and the Large Scale Structure
}

\author{
Toshiyuki Kanazawa \\ Department of Physics, University of Tokyo, Tokyo, 113-0033, Japan \\ M. Kawasaki \\ Research Center for the Early Universe (RESCEU), University of Tokyo, Tokyo, 113-0033, Japan \\ Naoshi Sugiyama \\ Department of Physics, Kyoto University, Kyoto, 606-8502 \\ T. Yanagida \\ Department of Physics and RESCEU, University of Tokyo, Tokyo 113-0033, Japan
}

(June 19, 2021)

\begin{abstract}
The cosmological implication of a double inflation model with hybrid + new inflations in supergravity is studied. The hybrid inflation drives an inflaton for new inflation close to the origin through supergravity effects and new inflation naturally occurs. If the total $e$-fold number of new inflation is smaller than $\sim 60$, both inflations produce cosmologically relevant density fluctuations. Both cluster abundances and galaxy distributions provide strong constraints on the parameters in the double inflation model assuming $\Omega_{0}=1$ standard cold dark matter scenario. The future satellite experiments to measure the angular power spectrum of the cosmic microwave background will make a precise determination of the model parameters possible.
\end{abstract}

Typeset using REVTEX 


\section{INTRODUCTION}

The idea of an inflationary universe [1,2] is very attractive since it can solve serious problems in standard big bang cosmology such as the horizon and flatness problems [1]. Though many types of inflation models have been proposed [3], there are basically three viable models: chaotic [4], new [5], and hybrid inflation [6]. These three models have their own characters. The chaotic inflation model is difficult to realize in the framework of supergravity since it requires a classical value of the inflaton field larger than the gravitational scale $\left(=2.4 \times 10^{18} \mathrm{GeV}\right.$ and it is taken to be unity throughout this paper). In supergravity the reheating temperature of inflation should be low enough to avoid overproduction of gravitinos [7,8]. The new inflation model [5] generally predicts a very low reheating temperature and hence it is the most attractive among the many inflation models. However, new inflation suffers from a fine-tuning problem about the initial condition; i.e., for successful new inflation, the initial value of the inflaton should be very close to the local maximum of the potential in a large region whose size is much longer than the horizon of the universe. On the other hand, hybrid inflation (and also the chaotic one) can occur for a large range of initial values.

Recently a framework of double inflation was proposed as a way to solve the initial value problem of the new inflation model [9]. It was shown that the above serious problem is solved by supergravity effects if there existed preinflation (e.g., hybrid inflation) with a sufficiently large Hubble parameter before new inflation [9]. Different models of double inflation were studied by various authors [14]. Unlike other double inflation scenarios, however, our double inflation is quite natural when we try to solve both the gravitino problem and the initial condition problem in the new inflation model.

In this double inflation model, if the $e$-fold number of the new inflation model is smaller than $\sim 60$, density fluctuations produced by both inflations are cosmologically relevant (the total $e$-fold number $\sim 60$ is required to solve flatness and horizon problems in standard big bang cosmology [10]). In this case, the preinflation should account for the density fluctuations on large cosmological scales [including the cosmic background explorer (COBE) scales], while the new inflation model produces density fluctuations on smaller scales. Although the amplitude of the fluctuations on large scales should be normalized to the COBE data [11, fluctuations on small scales are free from the COBE normalization and can have arbitrary power matched to the observation. In Refs. [12,13], the production of primordial black hole massive compact halo objects was considered in this double inflation model. In particular in Ref. [13], the coherent oscillation of the inflaton after a preinflation was taken into account.

In this paper, we study the cosmological implication of the double inflation model which induces a break on the cosmological ( $\gtrsim \mathrm{Mpc}$ ) scale in the initial density perturbations. It is well known that the observations of galaxy distributions cannot be accounted for with the cosmological density parameter $\Omega_{0}=1$ and the Hubble parameter $H_{0}=50 \mathrm{kms}^{-1} \mathrm{Mpc}^{-1}$ in a standard cold dark matter (CDM) model. However, in a double inflation case, there would be a possibility that the observations may be fit with $\Omega_{0}=1$ and without a cosmological constant, I since the produced density fluctuations would have a nontrivial shape. Rather

\footnotetext{
${ }^{1}$ Recently it was reported that the observations of a supernova type Ia suggests there is a nonzero
} 
we have a chance to determine parameters of double inflation by observations of the large scale structure of the universe. Taking hybrid inflation in supergravity [16] as an example of the preinflation, we find that the produced density fluctuations may account for the observed clusters abundances [17,18 and galaxy distributions 19 23 with $\Omega_{0}=1$ and $H_{0}=50 \mathrm{kms}^{-1} \mathrm{Mpc}^{-1}$.

\section{DOUBLE INFLATION MODEL}

We adopt the double inflation model proposed in Refs. [9, 12]. The model consists of two inflationary stages; the first one is called preinflation and we adopt hybrid inflation [16] as the preinflation. We also assume that the second inflationary stage is realized by a new inflation model [25] and its $e$-fold number is smaller than $\sim 60$. Thus, the density fluctuations on large scales are produced during the preinflation and their amplitude should be normalized to the COBE data [11]. On the other hand, the new inflation model produces fluctuations on small scales. Since the amplitude of the small scale fluctuations is free from the COBE normalization, we expect that the new inflation model can produce density fluctuations appropriate for the observations.

\section{A. Preinflation}

First, let us discuss the hybrid inflation model which we adopt to cause the preinflation. The hybrid inflation model contains two kinds of superfields: one is $S(x, \theta)$ and the others are $\Psi(x, \theta)$ and $\bar{\Psi}(x, \theta)$. Here $\theta$ is the Grassmann number denoting superspace. The model is based on the $\mathrm{U}(1)_{R}$ symmetry under which $S(\theta) \rightarrow e^{2 i \alpha} S\left(\theta e^{-i \alpha}\right)$ and $\Psi(\theta) \bar{\Psi}(\theta) \rightarrow \Psi\left(\theta e^{-i \alpha}\right) \bar{\Psi}\left(\theta e^{-i \alpha}\right)$. The superpotential is given by [6, 16]

$$
W(S, \Psi, \bar{\Psi})=-\mu^{2} S+\lambda S \bar{\Psi} \Psi
$$

The $R$-invariant Kähler potential is given by

$$
K(S, \Psi, \bar{\Psi})=|S|^{2}+|\Psi|^{2}+|\bar{\Psi}|^{2}-\frac{\zeta}{4}|S|^{4}+\cdots
$$

where $\zeta$ is a constant of order 1 and the ellipsis denotes higher-order terms, which we neglect in the present analysis. We gauge the $\mathrm{U}(1)$ phase rotation: $\Psi \rightarrow e^{i \delta} \Psi$ and $\bar{\Psi} \rightarrow e^{-i \delta} \bar{\Psi}$. To satisfy the $D$-term flatness condition we take always $\Psi=\bar{\Psi}$ in our analysis.

As is shown in Ref. [16] the real part of $S(x)$ is identified with the inflaton field $\sigma / \sqrt{2}$. The potential is minimized at $\Psi=\bar{\Psi}=0$ for $\sigma$ larger than $\sigma_{c}=\sqrt{2} \mu / \sqrt{\lambda}$ and inflation occurs for $0<\zeta<1$ and $\sigma_{c} \lesssim \sigma \lesssim 1$.

In a region of relatively small $\sigma\left(\sigma_{c} \lesssim \sigma \lesssim \lambda / \sqrt{8 \pi^{2} \zeta}\right)$ radiative corrections are important for the inflation dynamics as shown in Ref. [26]. Including one-loop corrections, the potential for the inflaton $\sigma$ is given by

cosmological constant $\left(\lambda_{0}\right)$. However, there are some papers which point out the problems of interpreting those observations [15]. 


$$
\begin{aligned}
V \simeq & \mu^{4}+\frac{1}{2} \zeta \mu^{4} \sigma^{2}+\frac{4 \zeta^{2}+7 \zeta+2}{16} \mu^{4} \sigma^{4} \\
& +\frac{\lambda^{2}}{128 \pi^{2}}\left[\left(\lambda \sigma^{2}-2 \mu^{2}\right)^{2} \ln \frac{\lambda \sigma^{2}-2 \mu^{2}}{\Lambda^{2}}\left(\lambda \sigma^{2}+2 \mu^{2}\right)^{2} \ln \frac{\lambda \sigma^{2}+2 \mu^{2}}{\Lambda^{2}}-2 \lambda^{2} \sigma^{4} \ln \frac{\lambda \sigma^{2}}{\Lambda^{2}}\right]
\end{aligned}
$$

where $\Lambda$ is a renormalization scale. The Hubble parameter $H_{\text {pre }}$ and $e$-fold number $N_{\text {pre }}$ are given by

$$
H_{\mathrm{pre}} \simeq \frac{\mu^{2}}{\sqrt{3}}
$$

and

$$
N_{\text {pre }}=\left|\int_{\sigma_{N_{\mathrm{pre}}}}^{\sigma_{c}} \frac{V}{V^{\prime}} d \sigma\right|,
$$

where $\sigma_{N_{\text {pre }}}$ is the value of the inflaton field $\sigma$ corresponding to an $e$-fold number $N_{\text {pre }}$.

If we define $N_{\mathrm{COBE}}$ as the $e$-fold number corresponding to the COBE scale, the COBE normalization leads to a condition for the inflaton potential:

$$
\frac{V^{3 / 2}\left(\sigma_{\mathrm{COBE}}\right)}{\left|V^{\prime}\left(\sigma_{\mathrm{COBE}}\right)\right|} \simeq 5.3 \times 10^{-4},
$$

where $\sigma_{\mathrm{COBE}} \equiv \sigma_{N_{\mathrm{COBE}}}$. In a hybrid inflation model, density fluctuation is almost scale free,

$$
n_{\text {pre }}=1+2\left(\frac{V^{\prime \prime}}{V}\right)-3\left(\frac{V^{\prime}}{V}\right)^{2} \simeq 1,
$$

where $n_{\text {pre }}$ is a spectral index for a power spectrum of density fluctuations.

\section{B. New inflation}

Now, we consider a new inflation model. We adopt the new inflation model proposed in Ref. [9]. The inflaton superfield $\phi(x, \theta)$ is assumed to have an $R$ charge $2 /(n+1)$ and $\mathrm{U}(1)_{R}$ is dynamically broken down to a discrete $Z_{2 n R}$ at a scale $v$, which generates an effective superpotential [9,25]:

$$
W(\phi)=v^{2} \phi-\frac{g}{n+1} \phi^{n+1}
$$

The $R$-invariant effective Kähler potential is given by

$$
K(\phi, \chi)=|\phi|^{2}+\frac{\kappa}{4}|\phi|^{4}+\cdots
$$

where $\kappa$ is a constant of order 1 .

The effective potential $V(\phi)$ for a scalar component of the superfield $\phi(x, \theta)$ in supergravity is obtained from the above superpotential (8) and the Kähler potential (9) as 


$$
V=e^{K(\phi)}\left\{\left(\frac{\partial^{2} K}{\partial \phi \partial \phi^{*}}\right)^{-1}\left|D_{\phi} W\right|^{2}-3|W|^{2}\right\},
$$

with

$$
D_{\phi} W=\frac{\partial W}{\partial \phi}+\frac{\partial K}{\partial \phi} W
$$

This potential yields a vacuum

$$
\langle\phi\rangle \simeq\left(\frac{v^{2}}{g}\right)^{1 / n} .
$$

In the true vacuum we have negative energy as

$$
\langle V\rangle \simeq-3 e^{\langle K\rangle}|\langle W\rangle|^{2} \simeq-3\left(\frac{n}{n+1}\right)^{2}|v|^{4}|\langle\phi\rangle|^{2}
$$

The negative vacuum energy (13) is assumed to be canceled out by a supersymmetry(SUSY)-breaking effect 25] which gives a positive contribution $\Lambda_{\text {SUSY }}^{4}$ to the vacuum energy. Thus, we have a relation between $v$ and the gravitino mass $m_{3 / 2}$ :

$$
m_{3 / 2} \simeq \frac{\Lambda_{\mathrm{SUSY}}^{2}}{\sqrt{3}}=\left(\frac{n}{n+1}\right)|v|^{2}\left|\frac{v^{2}}{g}\right|^{1 / n}
$$

The inflaton $\phi$ has a mass $m_{\phi}$ in the vacuum with (for details, see Ref. [25])

$$
m_{\phi} \simeq n|g|^{1 / n}|v|^{2-2 / n} .
$$

The inflaton $\phi$ may decay into ordinary particles through gravitationally suppressed interactions, which yields reheating temperature $T_{R}$ given by

$$
T_{R} \simeq 0.1 m_{\phi}^{3 / 2} \simeq 0.1 n^{3 / 2}|g|^{3 / 2 n}|v|^{3-3 / n}
$$

If we take $n=4$ and $g=1$,

$$
T_{R} \simeq 0.8|v|^{9 / 4}=1.9 \times 10^{18} \mathrm{GeV}\left(\frac{v}{M_{G}}\right)^{9 / 4} .
$$

In this case, the reheating temperature $T_{R}$ is as low as $2 \mathrm{GeV}-6 \times 10^{4} \mathrm{GeV}$ for $v \simeq 10^{-8}-10^{-6}$ $\left(m_{3 / 2} \simeq 0.02 \mathrm{GeV}-2 \mathrm{TeV}\right)$, for example, which is low enough to solve the gravitino problem.

Let us discuss dynamics of the new inflation model. Identifying the inflaton field $\varphi(x) / \sqrt{2}$ with the real part of the field $\phi(x)$, we obtain a potential of the inflaton for $\varphi<v$ from Eq. (10):

\footnotetext{
2 The decay rate of the inflaton $\phi$ is discussed in Ref. [9]
} 


$$
V(\varphi) \simeq v^{4}-\frac{\kappa}{2} v^{4} \varphi^{2}-\frac{g}{2^{\frac{n}{2}-1}} v^{2} \varphi^{n}+\frac{g^{2}}{2^{n}} \varphi^{2 n} .
$$

It has been shown in Ref. 225 that the slow-roll condition for the inflation is satisfied for $0<\kappa<1$ and $\varphi \lesssim \varphi_{f}$ where

$$
\varphi_{f} \simeq \sqrt{2}\left(\frac{(1-\kappa) v^{2}}{g n(n-1)}\right)^{1 /(n-2)} .
$$

New inflation ends when $\varphi$ becomes larger than $\varphi_{f}$. The Hubble parameter of the new inflation model is given by

$$
H_{\text {new }} \simeq \frac{v^{2}}{\sqrt{3}} .
$$

The $e$-fold number $N_{\text {new }}$ is given by

$$
N_{\text {new }}=\left|\int_{\varphi_{N_{\text {new }}}^{\varphi_{f}}}^{\varphi^{\prime}} \frac{V}{} d \varphi\right| .
$$

The amplitude of primordial density fluctuations $\delta \rho / \rho$ due to the new inflation model is written as

$$
\frac{\delta \rho}{\rho} \simeq \frac{1}{5 \sqrt{3} \pi} \frac{V^{3 / 2}\left(\varphi_{N_{\text {new }}}\right)}{\left|V^{\prime}\left(\varphi_{N_{\text {new }}}\right)\right|} \simeq \frac{1}{5 \sqrt{3} \pi} \frac{v^{2}}{\kappa \varphi_{N_{\text {new }}}} .
$$

Notice here that we have larger density fluctuations for smaller $\varphi_{N_{\text {new }}}$ and hence the largest amplitude of the fluctuations is given at the beginning of new inflation. An interesting point on the above density fluctuations is that it results in a tilted spectrum with spectral index $n_{\text {new }}$ given by (see Refs. [9,25])

$$
n_{\text {new }} \simeq 1-2 \kappa .
$$

\section{Initial value and fluctuations of $\varphi$}

The crucial point observed in Ref. [9] is that preinflation sets dynamically the initial condition for new inflation. The inflaton field $\varphi(x)$ for new inflation gets an effective mass $\sim \mu^{2}$ from the $e^{K}[\cdots]$ term in the potential (10) during preinflation [6,27]. Thus, we write the effective mass $m_{\text {eff }}$ as

$$
m_{\text {eff }}=c \mu^{2}=\sqrt{3} c H,
$$

where we introduce a free parameter $c$ since the precise value of the effective mass depends on the details of the Kähler potential. For example, if the Kähler potential contains $-f|\phi|^{2}|S|^{2}$, the effective mass is equal to $\sqrt{1+f} \mu^{2}$.

The evolution of the inflaton $\varphi$ for the new inflation model is described as

$$
\ddot{\varphi}+3 H \dot{\varphi}+m_{\mathrm{eff}}^{2} \varphi=0 .
$$


Using $\dot{H} \simeq 0$, we get a solution to the above equation as

$$
\varphi \propto a^{-3 / 2+\sqrt{9 / 4-3 c^{2}}}
$$

where $a$ denotes the scale factor of the universe. Thus, for $c \gtrsim \sqrt{3} / 2, \varphi$ oscillates during the preinflation and its amplitude decreases as $a^{-3 / 2}$. Thus, at the end of preinflation the $\varphi$ takes a value

$$
\varphi \simeq \varphi_{\min }+\left(\varphi_{i}-\varphi_{\min }\right) \exp \left(-\frac{3}{2} N_{\text {pre,tot }}\right)
$$

where $\varphi_{i}$ is the value of $\varphi$ at the beginning of preinflation, $\varphi_{\min }$ is the value of $\varphi$ at which the potential has a minimum, and $N_{\text {pre,tot }}$ is the total $e$-fold number of preinflation.

The $\varphi_{\text {min }}$ deviates from zero through the effect of the $\left|D_{S} W\right|^{2}+\left|D_{\phi} W\right|^{2}-3|W|^{2}$ term and the potential has a minimum [9] at

$$
\varphi_{\min } \simeq-\frac{\sqrt{2}}{c^{2} \sqrt{\lambda}} v\left(\frac{v}{\mu}\right)
$$

Thus, at the end of preinflation the $\varphi$ settles down to this $\varphi_{\min }$.

After preinflation, the $\sigma$ and $\Psi(\bar{\Psi})$ start to oscillate and the universe becomes matter dominated. $\Psi$ and $\bar{\Psi}$ couple to the $U(1)$ gauge multiplets and decay immediately to gauge fields if energetically allowed. We assume that masses for the gauge fields are larger than those of $\Psi$ and $\bar{\Psi}$. We also assume that the supersymmetric (SUSY) standard model particles do not couple to the gauge multiplets. Thus, $S, \Psi$, and $\bar{\Psi}$ decay into light particles only through gravitationally suppressed interactions and the coherent oscillations of $S$, $\Psi$, and $\bar{\Psi}$ fields continue until new inflation starts. In this period of the coherent oscillations the average potential energy of the scalar fields is the half of the total energy of the universe and hence the effective mass of $\varphi$ is given by

$$
m_{\mathrm{eff}}^{2} \simeq \frac{3}{2} H^{2}
$$

Here and hereafter, we take $c=1$. The evolution of $\varphi$ is described by Eq. (25). Taking into account $\dot{H}=(3 / 2) H^{2}$, one can find that the amplitude of $\varphi$ decreases as $a^{-3 / 4}$. After the preinflation ends, the superpotential for the inflaton of the preinflation vanishes and hence the potential for $\varphi$ has a minimum at $\varphi \simeq 0$.

During the matter-dominated era between two inflations, the energy density scales as $\propto a^{-3}$, and it is $\mu^{4}$ and $v^{4}$ for a hybrid inflation and a new inflation, respectively, the scale factor increases by a factor $(\mu / v)^{4 / 3}$ during this era. Thus, the mean initial value $\varphi_{b}$ of $\varphi$ at the beginning of new inflation is written as

$$
\varphi_{b} \simeq \frac{\sqrt{2}}{\sqrt{\lambda}} v\left(\frac{v}{\mu}\right)^{2}\left[\cos \left(\sqrt{\frac{5}{3}} \ln \frac{\mu}{v}\right)+\sqrt{\frac{3}{5}} \sin \left(\sqrt{\frac{5}{3}} \ln \frac{\mu}{v}\right)\right] .
$$

\footnotetext{
${ }^{3}$ Here we have assumed that when $\varphi$ begins oscillating just after the preinflation, the time derivative of it vanishes.
} 
We now discuss quantum effects during preinflation. It is known that in a de Sitter universe massless fields have quantum fluctuations whose amplitudes are given by $H /(2 \pi)$. However, the quantum fluctuations for $\varphi$ are strongly suppressed [28] in the present model since the mass of $\varphi$ is larger than the Hubble parameter until the start of new inflation.

Let us consider the amplitude of fluctuations with comoving wave number $k_{b}$ corresponding to the horizon scale at the beginning of new inflation. These fluctuations are induced during preinflation and its amplitude at horizon crossing $\left[k_{b}=a\left(t_{h}\right) H_{\text {pre }}\right.$, where $t_{h}$ is a time of horizon crossing] is given by $H_{\text {pre }} /(2 \pi)\left(H_{\text {pre }} / m_{\text {eff }}\right)^{1 / 2}$. f

Since those fluctuations reenter the horizon at the beginning of new inflation $\left(t=t_{b}\right)$, the scale factor of the universe increases from $t_{h}$ to $t_{b}$ by a factor of $\left(H_{\text {pre }} / H_{\text {new }}\right)=(\mu / v)^{2}$. As we have seen above, the amplitude of fluctuations decreases as $a^{-3 / 2}$ during preinflation and $a^{-3 / 4}$ during the matter-dominated era between two inflations. Since the scale factor increases by $(\mu / v)^{4 / 3}$ during the matter-dominated era and by $(\mu / v)^{2}$ from $t_{h}$ to $t_{b}$, respectively, it increases by $(\mu / v)^{2 / 3}$ from $t_{h}$ to the beginning of the matter-dominated era. Therefore, the amplitude of fluctuations with comoving wavelength corresponding to the horizon scale at the beginning of new inflation is now given by

$$
\delta \varphi \simeq \frac{H_{\mathrm{pre}}}{2 \pi}\left(\frac{H_{\mathrm{pre}}}{m_{\mathrm{eff}}}\right)^{1 / 2}\left[\left(\frac{\mu}{v}\right)^{2 / 3}\right]^{-3 / 2}\left[\left(\frac{\mu}{v}\right)^{4 / 3}\right]^{-3 / 4} \simeq \frac{H_{\mathrm{pre}}}{3^{1 / 4} 2 \pi}\left(\frac{v}{\mu}\right)^{2} .
$$

The fluctuations given by Eq. (31) are a little less than newly induced fluctuations at the beginning of new inflation $\left[\simeq v^{2} /(2 \pi \sqrt{3})\right]$. Moreover, the fluctuations produced during preinflation are more suppressed for smaller wavelength. Thus, we assume that the fluctuations of $\varphi$ induced in preinflation can be neglected when we estimate the fluctuations during new inflation.

Here let us estimate the $e$-fold number which corresponds to our current horizon. From Eq.(16), the reheating temperature after new inflation is given as

$$
T_{R} \simeq 0.1 m_{\phi}^{3 / 2} \simeq 0.1 n^{3 / 2}|g|^{3 / 2 n}|v|^{3-3 / n} \simeq 0.8|v|^{9 / 4}
$$

Here and hereafter we take $n=4$ and $g=1$ for simplicity. The $e$-fold number is given by 29

$$
N_{\text {tot }}=62-\ln \frac{k}{a_{0} H_{0}}-\ln \frac{10^{16} \mathrm{GeV}}{V^{1 / 4}}+\ln \frac{V^{1 / 4}}{V_{\text {end }}^{1 / 4}}-\frac{1}{3} \ln \frac{V_{\text {end }}^{1 / 4}}{\rho_{\text {reh }}^{1 / 4}}
$$

where $V$ is a potential energy when a given scale $k$ leaves the horizon, $V_{\text {end }}$ is when the inflation ends, and $\rho_{\text {reh }}$ is the energy density at the time of reheating. Now we can take $V \simeq V_{\text {end }}$, and $\rho_{\text {reh }}^{1 / 4} \simeq$ a few $\times T_{\text {reh. }}$. Therefore, for $k=a_{0} H_{0}$ (i.e., present horizon scale),

$$
N_{\text {tot }} \simeq 67.8+\frac{17}{12} \ln v .
$$

\footnotetext{
${ }^{4}$ This is valid when $m_{\text {eff }}$ is greater than $3 H_{\text {pre }} / 2$.
} 
Since preinflation lasts after the scale $k_{b}$ crosses the horizon by an $e$-folding number $\ln (\mu / v)^{2 / 3}=(2 / 3) \ln (\mu / v)$ as mentioned above, the $e$-folding number corresponding to the COBE scale can be expressed as

$$
\begin{aligned}
N_{\mathrm{COBE}} & =N_{\text {tot }}-N_{\text {new }}+\frac{2}{3} \ln \frac{\mu}{v} \\
& \simeq 67.8+\frac{17}{12} \ln v-N_{\text {new }}+\frac{2}{3} \ln \frac{\mu}{v},
\end{aligned}
$$

when we consider COBE normalization, Eq. (6), we have to use this quantity.

Finally, we make a comment on the domain-wall problem in the double inflation model. Since the potential of the inflaton $\phi$ has a discrete symmetry [see Eqs. (8) and (9)], domain walls are produced if the phases of $\phi$ are spatially random. However, preinflation makes the phase of $\phi$ homogeneous with the help of the interactions between two inflaton fields $S$ and $\phi$ [see Eq. (28)]. Therefore, the domain-wall problem does not exist in the present model.

\section{Numerical results}

We estimate density fluctuations in the double inflation model numerically by calculating the evolution of $\varphi$ and $\sigma$. For simplicity, we take $\zeta=0$.

Since we are concerned with the situation where the breaking (transit scale from the hybrid inflation to the new inflation) occurs at cosmological scale, we choose a parameter region in which the breaking scale comes within the range

$$
10^{-3} h \mathrm{Mpc}^{-1} \lesssim k_{b} \lesssim 1 h \mathrm{Mpc}^{-1}
$$

where $h=H_{0} /\left(100 \mathrm{kms}^{-1} \mathrm{Mpc}^{-1}\right)$ and it takes $h=0.5$ in this paper. Also, we require that the ratio between the density fluctuation produced by a hybrid inflation and that by new inflation is

$$
0.1 \lesssim \mathcal{R} \equiv \frac{P_{\text {new }}}{P_{\text {pre }}} \lesssim 10
$$

where $P_{\text {new }}$ and $P_{\text {pre }}$ refer to the amplitude of a power spectrum of density fluctuations at $k_{b}$, produced by new inflation and preinflation, respectively:

$$
P(k)=\left\{\begin{array}{l}
P_{\text {pre }}\left(\frac{k}{k_{b}}\right)^{1} T^{2}(k) \quad\left(k<k_{b}\right), \\
P_{\text {new }}\left(\frac{k}{k_{b}}\right)^{n_{\text {new }}} T^{2}(k)=P_{\text {pre }} \mathcal{R}\left(\frac{k}{k_{b}}\right)^{n_{\text {new }}} T^{2}(k) \quad\left(k>k_{b}\right),
\end{array}\right.
$$

where $T(k)$ is a CDM transfer function. We draw a sample of results in Fig. 10 for $v=10^{-7}$. From this figure we can see that if

$$
\lambda \sim \mathcal{O}\left(10^{-4}-10^{-3}\right) \text { and } 0.1 \lesssim \kappa \lesssim 0.2
$$

$k_{b}$ is at a cosmological scale, and density fluctuations produced during new inflation are not too far from that of preinflation. We can understand the qualitative dependence of $\left(k_{b}, \mathcal{R}\right)$ on $(\kappa, \lambda)$ as follows: When $\kappa$ is large, the slope of the potential for new inflation is too steep, 
and new inflation cannot last for a long time. Therefore, the break occurs at smaller scales. As for $\mathcal{R}$, we can see that the larger $\lambda$ is, the larger $\mu$ is, from Eq.(6). In addition, from Eqs. 22) and (30), we can see that

$$
\left(\frac{\delta \rho}{\rho}\right)_{\text {new }} \propto \frac{1}{\kappa \varphi_{\mathrm{N}_{\mathrm{new}}}} \sim \frac{\sqrt{\lambda} \mu^{2}}{\kappa}
$$

for a fixed $v$. Thus, we have larger $\mathcal{R}$ for larger $\lambda$.

\section{COMPARISON WITH OBSERVATIONS}

In this section we compare the result of our double inflation model with the observations of the cluster abundances [17,18] and galaxy distributions [19 23].

\section{A. Cluster Abundances}

Since the power spectrum of density fluctuations shows a break on the cosmological scale in this double inflation model, we cannot simply employ the value of $\sigma_{8}$ quoted by previous works [17,18]. We need to calculate the cluster abundances by using the PressSchechter theory [30]. According to the Press-Schechter theory, the comoving number density of collapsed systems of mass $M$ at redshift $z$, per interval $d M$, is expressed as

$$
\frac{d n(M, z)}{d M}=\sqrt{\frac{2}{\pi}} \frac{\rho}{M} \frac{\delta_{c}(z)}{\sigma^{2}(M)}\left|\frac{d \sigma(M)}{d M}\right| \exp \left[-\frac{\delta_{c}^{2}(z)}{2 \sigma^{2}(M)}\right]
$$

where $\rho$ is the mean mass density of the universe at present, and $\sigma(M)$ is the mass variance, the rms density fluctuations smoothed over the mass scale $M$, which is defined as

$$
\sigma^{2}(M) \equiv \frac{1}{(2 \pi)^{3}} \int P(k ; A) W^{2}\left(k r_{0}\right) d^{3} k
$$

where $M=4 \pi r_{0}^{3} \rho / 3, W\left(k r_{0}\right)$ is a window function

$$
W\left(k r_{0}\right)=\frac{3}{\left(k r_{0}\right)^{3}}\left[\sin \left(k r_{0}\right)-k r_{0} \cos \left(k r_{0}\right)\right],
$$

and $P(k ; A)$ is a present matter density fluctuation power spectrum with a normalization $A$. For the case of $\Omega_{0}=1, \delta_{c}(z)=1.686(1+z)$. This is the density contrast that a collapsed region should have at collapse time if it had always evolved according to linear theory. In this paper we take total matter density $\Omega_{0}=1$ and use the observations of neighbor clusters $(z \simeq 0)$.

Given the power spectrum, we can obtain the cluster abundance from the Press-Schechter theory. When we determine the breaking scale $k_{b}$, the power spectrum ratio $\mathcal{R} \equiv P_{\text {new }} / P_{\text {pre }}$, and the spectral index for new inflation $n_{\text {new }}$, we can get the power spectrum up to normalization $A_{\mathrm{cl}}$. Using this power spectrum we can calculate the mass variance and obtain, from Eq.(41), 


$$
n\left(>M_{\min } ; A_{\mathrm{cl}}\right)=\int_{M_{\min }}^{\infty} \frac{d n(M)}{d M} d M .
$$

Many clusters of galaxies are observed using x-ray fluxes. Under the assumption that clusters are hydrostatic, we can obtain the mass-temperature relations as

$$
T_{\text {gas }}=\frac{9.37 \mathrm{keV}}{\beta(5 X+3)}\left(\frac{M}{10^{15} h^{-1} M_{\odot}}\right)^{2 / 3}(1+z)\left(\frac{\Omega_{0}}{\Omega(z)}\right)^{1 / 3} \Delta_{c}^{1 / 3},
$$

where $\Delta_{c}$ is the ratio of the mean density of a cluster to the critical density at that redshift, $\beta$ is the ratio of specific galaxy kinetic energy to specific gas thermal energy, and $X$ is the hydrogen mass fraction. We take $X=0.76, \beta=1$, and $\Delta_{c}=18 \pi^{2} \simeq 178$ [17]. Then Eq. (45) reduces to

$$
T_{\text {gas }} \simeq 7.75\left(\frac{M}{10^{15} h^{-1} M_{\odot}}\right)^{2 / 3} \mathrm{keV} .
$$

The observed cluster abundance as a function of x-ray temperature can be translated into a function of mass using Eq. (45). Accumulating the observations, Henry and Arnaud [31] gave the fitting formula as

$$
\frac{d n(T)}{d T}=1.8\left\{\begin{array}{c}
+0.8 \\
-0.5
\end{array}\right\} \times 10^{-3} h^{3} \mathrm{Mpc}^{-3} \mathrm{keV}^{-1}\left(\frac{k T}{1 \mathrm{keV}}\right)^{-4.7 \pm 0.5} .
$$

By integrating Eq. (47) we obtain

$$
3.1 \times 10^{-4}\left(\frac{T_{\min }}{\mathrm{keV}}\right)^{-4.2} \lesssim n\left[>M_{\min }=M\left(T_{\min }\right)\right] \lesssim 8.1 \times 10^{-4}\left(\frac{T_{\min }}{\mathrm{keV}}\right)^{-3.2},
$$

where the unit of cluster abundance is $h^{3} \mathrm{Mpc}^{-3}$. Henry and Arnaud 31 also gave a table of cluster observations whose temperatures are larger than $2.5 \mathrm{keV}$, which corresponds to a lower limit $M_{\min }=1.8 \times 10^{14} h^{-1} M_{\odot}$ [see Eq.(46)]. Therefore we have, from Eq. (48),

$$
6.6 \times 10^{-6} \lesssim n\left(>1.8 \times 10^{14} h^{-1} M_{\odot}\right) \lesssim 4.3 \times 10^{-5} .
$$

Matching these abundances, Eq. (44) calculated from the Press-Schechter theory, and Eq. (49) inferred from the x-ray cluster observations, we can determine the normalization (amplitude) of power spectrum, $A_{\mathrm{cl}}$. Using this normalization, we can obtain "cluster abundance normalized" $\sigma_{8}, \sigma_{8, \mathrm{cl}}$, as

$$
\left.\sigma_{8, \mathrm{cl}}^{2} \equiv \int_{0}^{\infty} \frac{k^{3}}{2 \pi^{2}} P\left(k ; A_{\mathrm{cl}}\right) W^{2}\left(k r_{0}\right) \frac{d k}{k}\right|_{r_{0}=8 h^{-1} \mathrm{Mpc}} .
$$

Because of error bars, we have a range of $\sigma_{8, \mathrm{cl}}$ from observations. On the other hand, we can normalize the power spectrum by COBE data [11,32]. Therefore, we have "COBE normalized" $\sigma_{8}, \sigma_{8, \mathrm{COBE}}$ together with $\sigma_{8, \mathrm{cl}}$. Bunn and White [32] estimates one standard deviation error of COBE normalization to be $7 \%$ which is much smaller than the one of cluster normalization. We conclude that if $\sigma_{8, \mathrm{COBE}}$ lies in a $\sigma_{8, \mathrm{cl}}$ range, that the parameter region of $k_{b}, \mathcal{R}$, and $n_{\text {new }}$ is consistent with the cluster abundance observations (see lightly shaded region of Fig. 3 for $v=10^{-7}$ ). 


\section{B. galaxy distributions}

There are many observations which measure the density fluctuations from galaxy distributions. Among these we use following data sets in this paper.

- Southern Sky Redshift Survey of optically selected galaxies (SSRS2) \& The Center for Astrophysics redshift survey of the northern hemisphere (CfA2) (101Mpc/h volumelimited, $\left.M_{B}<-19.7+5 \log h\right)$, analyzed by da Costa et al [19].

- The same with above $\left(130 \mathrm{Mpc} / h\right.$ volume-limited, $\left.M_{B}<-20.3+5 \log h\right) 19$.

- The Las Campanas Redshift Survey (LCRS), analyzed by Lin et al [20].

- The Infrared Astronomical Satellite (IRAS) 1.2 Jy Sample, analyzed by Fisher et al [21].

- The Queen Mary College, Durham, Oxford, and Toronto (QDOT) survey, analyzed by Feldman et al [22].

- IRAS $1.2 \mathrm{Jy}+$ QDOT $[P(k)=8000$ weighting], analyzed by Tadros and Efstathiou [23].

These data are compiled by Vogeley [24]. In Fig. 2, we plot the observations we use in this paper.

Employing the COBE normalization, we can determine the power spectrum with its overall amplitude if we fix the the breaking scale $k_{b}$, the power spectrum ratio $\mathcal{R} \equiv P_{\text {new }} / P_{\text {pre }}$, and the spectral index for new inflation $n_{\text {new }}$. One might want to make direct comparison of this power spectrum with above observations of galaxy distributions. However, distribution of luminous objects such as galaxies could differ from underlying mass distribution because of so-called bias. There is even no guarantee that each observational sample has same bias factor. Therefore, we only consider the shape of the power spectrum here. We change the overall amplitude of each set of observations arbitrarily. And we estimate the goodness of fitting by calculating $\chi^{2}$ of this power spectrum with fixing $k_{b}, \mathcal{R}$, and $n_{\text {new }}$.

In Fig. 3, we plot a sample of our results for $v=10^{-7}$. There is a parameter region where both the cluster abundances and galaxy distributions can be accounted for by our model. What we can see from this figure is that we have almost fixed value of $\kappa$ and $\lambda$, if we require that a break should occur at a cosmological scale. The results for $v=10^{-6.5}$ to $v=10^{-7.5}$ are summarized in Table \& (outside of this range, we cannot find a suitable parameter region), where we write the coupling constants $\kappa$ and $\lambda$.

In Fig. 4 , we plot the power spectrum for a standard CDM model with the optimized galaxy distributions. In this case, $\chi^{2}$ normalized by the degree of freedom is 1.74 . Also in Fig.5, we plot the power spectrum for one of the parameters which minimize $\chi^{2}(\mathcal{R}=$ $\left.0.345, n_{\text {new }}=0.8, k_{b}=0.023746 h^{-1} \mathrm{Mpc}\right)$, where the $\chi^{2}$ normalized by the d.o.f. is 1.07 .

\section{CMB anisotropies}

In our double inflation model, the density fluctuations at smaller scales are produced during new inflation, and they have different amplitudes from COBE normalization. Also, 
they are tilted in general $\left(n_{\text {new }}=1-2 \kappa<1\right)$. Thus, the cosmic microwave background (CMB) anisotropy angular power spectrum would have a nontrivial shape at smaller scales. We choose nine parameter sets from Fig. 3 [see TableIT], and calculate the CMB angular power spectra (Fig.6). In the allowed parameter region, $\mathcal{R} \lesssim 1$ and $n_{\text {new }}<1$. In this region, the characteristic feature known as the acoustic peaks is suppressed compared with the standard CDM case (dashed lines). Some of them show a dip on the scales, which correspond to the breaking scales $k_{b}$, larger than the first peak (smaller in $\ell$ ). Although such recent medium angle experiments as Saskatoon [33], QMAP [34], and TOCO97/TOCO98 [35] have reported the existence of the first acoustic peak, these results are inconclusive in view of rather large observational errors. The observations of CMB anisotropies by future satellite experiments [Microwave Anisotropy Probe (MAP) [36], Planck [37]] would be able to test our models.

\section{CONCLUSIONS AND DISCUSSIONS}

In this paper we have studied the density fluctuations produced in the double inflation model in supergravity, and compared it to the observations. Our double inflation model consists of preinflation (= hybrid inflation ) and new inflation. Preinflation provides the density fluctuations observed by COBE and it also dynamically sets the initial condition of new inflation through supergravity effects. The predicted power spectrum has almost a scale-invariant form $\left(n_{s} \simeq 1\right)$ on large cosmological scales which is favored for the structure formation of the universe [38]. On the other hand, new inflation gives the power spectrum which has different amplitude and shallow slope $\left(n_{s}<1\right)$ on small scales. Thus, this power spectrum has a break on the scale corresponding to the turning epoch from preinflation to new inflation.

We have shown that there is a parameter region where the double inflation model produces an appropriate power spectrum, i.e., the break occurs at a cosmological scale and both cluster abundances and galaxy distributions can be accounted for.

We have also calculated the CMB angular power spectra for some appropriate parameters. In our double inflation model, the acoustic peaks are suppressed compared with the no-break model. Future satellite experiments would be able to test our model and will make precise determination of model parameters possible.

\section{ACKNOWLEDGMENT}

The authors thank M. Vogeley for kindly providing the data for galaxy distributions. T. K. is grateful to K. Sato for his continuous encouragement, and to T. Kitayama for useful discussions. N.S. thanks the Max Planck Institute for Astrophysics in Garching for

their warm hospitality. A part of work is supported by Grant-in-Aid from the Ministry of Education. 


\section{REFERENCES}

[1] A. H. Guth, Phys. Rev. D23, 347 (1981).

[2] K. Sato, Mon. Not. R. Astron. Soc. 195, 467 (1981); A. A. Starobinsky, Pis'ma Zh. Éksp. Teor. Fiz. 30, 719 81979) [JETP Lett. 30, 682 (1979)]; Phys. Lett. B91, 99 (1980).

[3] For example, A.D. Linde, Particle Physics and Inflationary Cosmology (Harwood, Chur, Switzerland, 1990).

[4] A. D. Linde, Phys. Lett. B129, 177 (1983).

[5] A. Albrecht and P.J. Steinhardt, Phys. Rev. Lett. 48, 1220 (1982); A.D. Linde, Phys. Lett. B108, 389 (1982).

[6] A. D. Linde, Phys. Rev. D49, 748 (1994); E. J. Copeland, A. R. Liddle, D. H. Lyth, E. D. Stewart, and D. Wands, ibid. D49, 6410 (1994).

[7] M. Yu. Khlopov and A.D. Linde, Phys. Lett. B138, 265 (1984) ; J. Ellis, E. Kim and D.V. Nanopoulos, ibid.B145, 181 (1984); J. Ellis, G.B. Gelmini, J.L. Lopez, D.V. Nanopoulos, and S. Sarker, Nucl. Phys. B373, 399 (1992); M. Kawasaki and T. Moroi, Prog. Theor. Phys. 93, 879 (1995).

[8] T. Moroi, H. Murayama, and M. Yamaguchi, Phys. Lett. B303, 289 (1993).

[9] K.I. Izawa, M. Kawasaki, and T. Yanagida, Phys. Lett. B411, 249 (1997).

[10] E. W. Kolb and M. S. Turner, The Early Universe (Addison-Wesley, Reading MA, 1990).

[11] C.L. Bennett et al., Astrophys. J. 464, L1 (1996).

[12] M. Kawasaki, N. Sugiyama, and T. Yanagida, Phys. Rev. D57, 6050 (1998).

[13] M. Kawasaki and T. Yanagida, Phys. Rev. D59, 043512 (1999).

[14] L.A. Kofman, A.D. Linde, and A.A. Starobinsky, Phys. Lett. B 157, 361 (1985); J. Silk and M.S. Turner, Phys. Rev. D 35, 419 (1987); D. Polarski and A.A. Starobinsky, Nucl. Phys. B 385, 623 (1992);

P. Peter, D. Polarski, and A.A. Starobinsky, Phys. Rev. D 50, 4827 (1994); S. Gottlöber, J. P. Mücket, and A. A. Starobinsky, Astrophys. J. 434, 417 (1994); R. Kates, V. Müller, S. Gottlöber, J. P. Mücket, and J. Retzlaff, Mon. Not. Roy. Astron. Soc. 277, 1254 (1995); J.A. Adams, G.G. Ross, and S. Sarkar, Nucl. Phys. B 503, 405 (1997); J. Lesgourgues and D. Polarski, Phys. Rev. D 56, 6425 (1997); J. Lesgourgues, D. Polarski and A.A. Starobinsky, Mon. Not. Roy. Astron. Soc. 297, 769 (1998); M. Sakellariadou and N. Tetradis, hep-ph/9806461.

[15] I. Dominguez, P. Hoeflich, O. Straniero, and C. Wheeler, astro-ph/9905047; P. S. Drell, T. J. Loredo, and I. Wasserman, astro-ph/9905027; A. N. Aguirre, astro-ph/9904319;

A. G. Riess, A. V. Filippenko, W. Li and B. P. Schmidt, astro-ph/9907038.

[16] C. Panagiotakopoulous, Phys. Rev. D55, 7335 (1997); A. Linde and A. Riotto, ibid.56, R1841 (1997).

[17] V. Eke, S. Cole, and C. S. Frenk, Mon. Not. R. Astron. Soc. 282, 263 (1996).

[18] P. T. P. Viana and A. R. Liddle, in Proceedings of the Conference "Cosmological Constraints from X-Ray Clusters", astro-ph/9902245.

[19] L. N. da Costa et al., Astrophys. J. 437, L1 (1994).

[20] H. Lin et al., Astrophys. J. 471, 617 (1996).

[21] K. B. Fisher et al., Astrophys. J. 402, 42 (1993).

[22] H. A. Feldman, N. Kaiser, and J. A. Peacock, Astrophys. J. 426, 23 (1994). 
[23] H. Tadros and G. Efstathiou, Mon. Not. R. Astron. Soc. 276, L45 (1995).

[24] M. S. Vogeley, in The Evolving Universe, edited by D. Hamilton (Kluwer, Dordrecht, 1998), p.395.

[25] K.I. Izawa and T. Yanagida, Phys. Lett. B393, 331 (1997).

[26] G. Dvali, Q. Shafi, and R.K. Shaefer, Phys. Rev. Lett. 73, 1886 (1994).

[27] K. Kumekawa, T. Moroi, and T. Yanagida, Prog. Theor. Phys. 92, 437 (1994); M. Dine, L. Randall, and S. Thomas, Phys. Rev. Lett. 75, 398 (1995).

[28] K. Enquvist, K.W. Ng, and K.A. Olive, Nucl. Phys. B303, 713 (1988).

[29] A. R. Liddle and D. H. Lyth, Phys. Rep. 231, 1 (1995).

[30] W. H. Press and P. Schechter, Astrophys. J. 187, 425 (1974).

[31] J. P. Henry and K. A. Arnaud, Astrophys. J. 372, 410 (1991).

[32] E. F. Bunn and M. White, Astrophys. J. 480, 6 (1997)

[33] C. B. Netterfield et al., Astrophys. J. 474, 47 (1997).

[34] A. de Oliveira-Costa et al., Astrophys. J. 509, L77 (1998).

[35] E. Torbet et al., Astrophys. J. 521, L79 (1999); A. D. Miller et al., astro-ph/9906421.

[36] http://map.gsfc.nasa.gov

[37] http://astro.estec.esa.nl/SA-general/Projects/Planck/

[38] M. White, D. Scott, J. Silk, and M. Davis, Mon. Not. R. Astron. Soc. 276, L69 (1995). 


\section{TABLES}

TABLE I. $\kappa$ and $\lambda$ for each $v$

\begin{tabular}{|c||cc|}
\hline \hline$v$ & $\kappa$ & $\lambda$ \\
\hline $10^{-6.5}$ & $0.03 \sim 0.05$ & $1.8 \times 10^{-4} \sim 2 \times 10^{-4}$ \\
$10^{-6.8}$ & $0.075 \sim 0.085$ & $1.5 \times 10^{-4} \sim 3 \times 10^{-4}$ \\
$10^{-7}$ & $0.09 \sim 0.11$ & $2 \times 10^{-4} \sim 3 \times 10^{-4}$ \\
$10^{-7.3}$ & $0.125 \sim 0.14$ & $2 \times 10^{-4} \sim 2.5 \times 10^{-4}$ \\
$10^{-7.5}$ & $0.145 \sim 0.16$ & $1.5 \times 10^{-4} \sim 2 \times 10^{-4}$ \\
\hline \hline
\end{tabular}

TABLE II. nine parameter sets.

\begin{tabular}{|c||cccc|}
\hline \hline$n_{\text {new }}$ & $\kappa$ & $\lambda \times 10^{4}$ & $k_{b}\left(h^{-1} \mathrm{Mpc}\right)$ & $\mathcal{R}$ \\
\hline \multirow{3}{*}{0.78} & & 2.9 & 0.029004 & 0.532 \\
& 0.109 & 3.1 & 0.007152 & 0.7112 \\
& & 3.344 & 0.001596 & 01.002 \\
\hline \multirow{3}{*}{0.79} & \multirow{3}{*}{0.104} & 2.7 & 0.019442 & 0.437 \\
& & 2.878 & 0.005298 & 0.570 \\
& & 3.056 & 0.001444 & 0.738 \\
\hline \multirow{3}{*}{0.80} & \multirow{3}{*}{0.098} & 2.456 & 0.013032 & 0.340 \\
& & 2.589 & 0.004338 & 0.418 \\
& & 2.7 & 0.001949 & 0.495 \\
\hline \hline
\end{tabular}




\section{FIGURES}

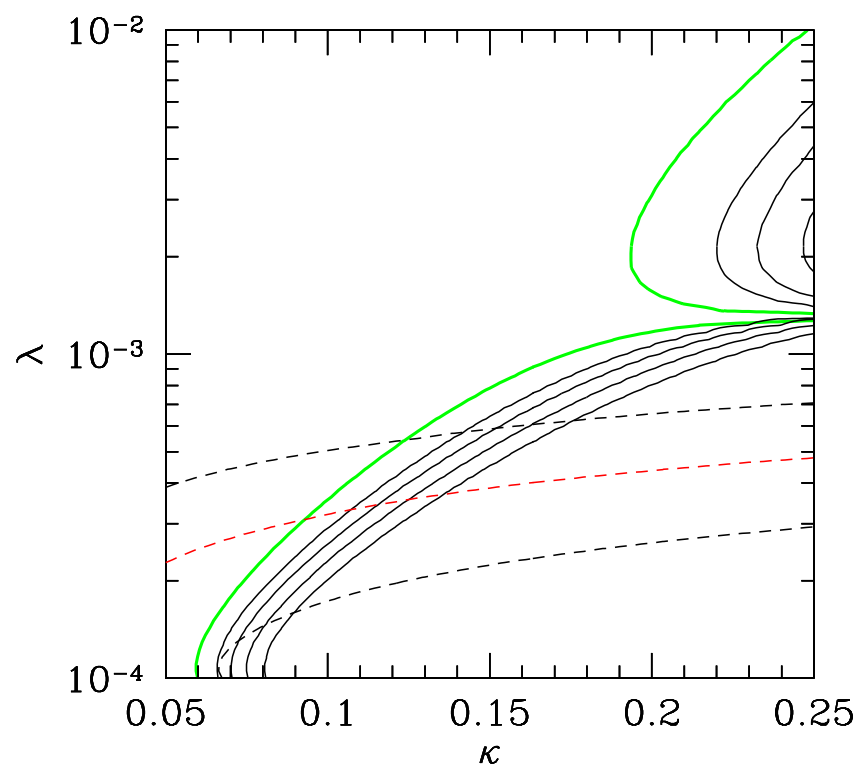

FIG. 1. A double inflation result for $v=10^{-7}$. The solid lines correspond to the breaking scale $k_{b}=10^{-3}, 10^{-2}, 10^{-1}$ and $1 h \mathrm{Mpc}^{-1}$, from left to right. Three dashed lines represent power spectra ratio $\mathcal{R}=10,1$, and 0.1 , from top to bottom. The region on the left hand side of the thick solid line is irrelevant since COBE scale fluctuations are produced during new inflation.

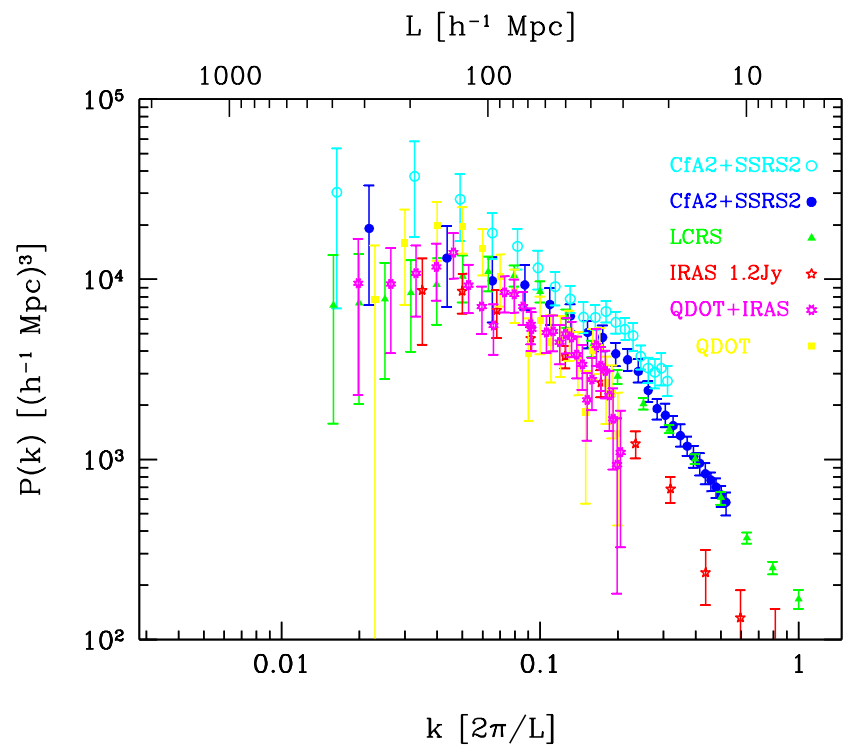

FIG. 2. The observations we have used in this paper to constrain our double inflation model. Each symbol represents observations (see text, figure courtesy of M. Vogeley). 


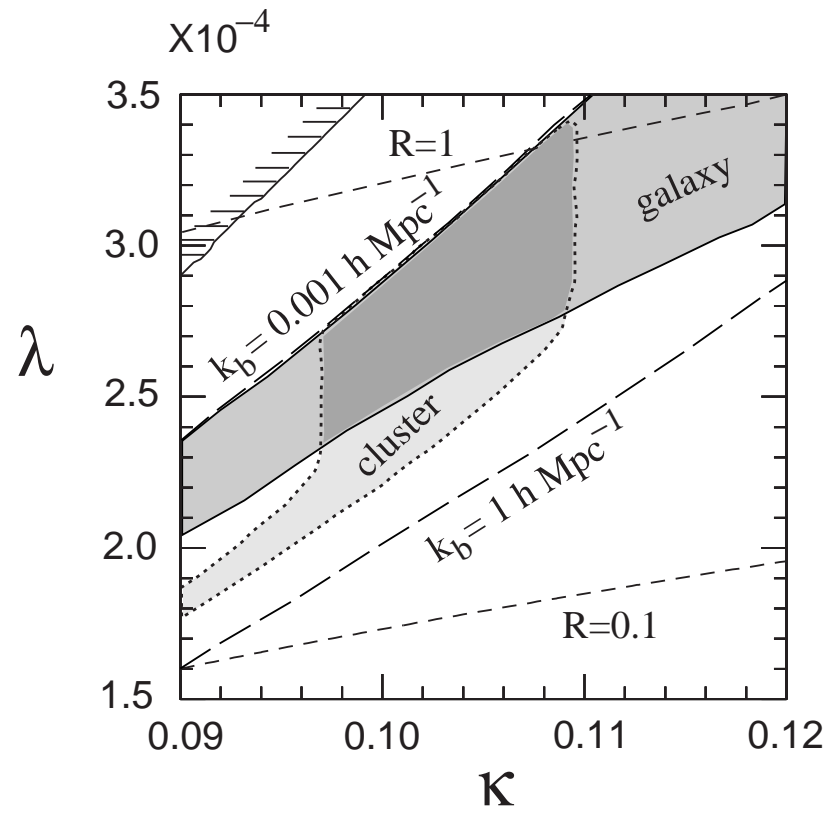

FIG. 3. The allowed parameter region for $v=10^{-7}$. The region on a top-left corner is irrelevant since the COBE scale fluctuation is produced during new inflation. The lightly shaded region inside the dotted line (named "cluster") indicates a region where "cluster normalized" and "COBE normalized" $\sigma_{8}$ are consistent. The darkly shaded region satisfies the constraints from both the cluster abundances and the galaxy distributions (99\% C.L. $\chi^{2}$ fitting). $\mathcal{R}$ is a power spectra ratio.

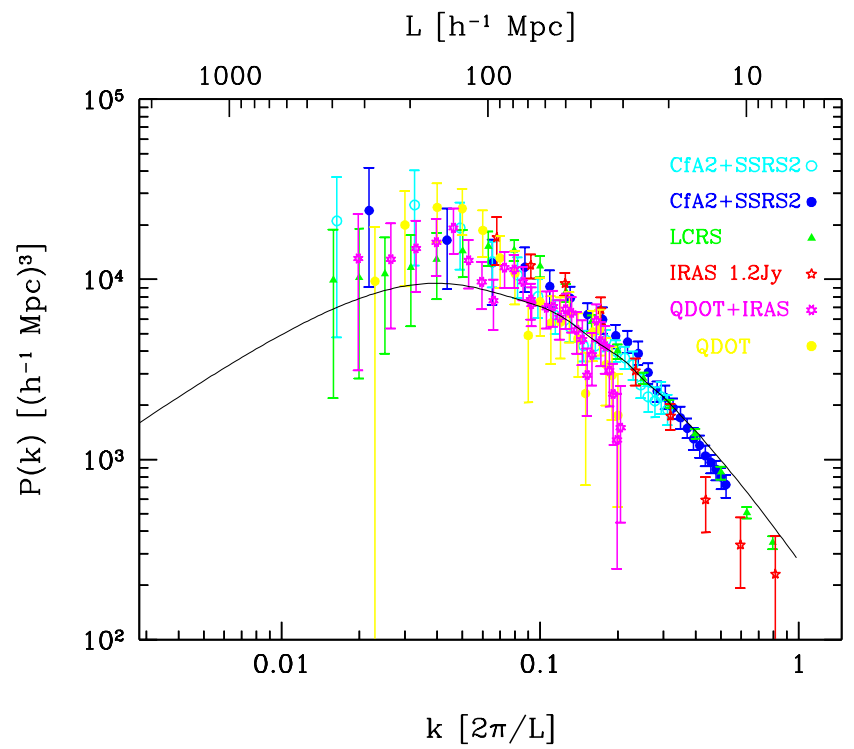

FIG. 4. The power spectrum with optimized galaxy distributions for a standard CDM. $\chi^{2} /$ d.o.f. $=1.74$. 


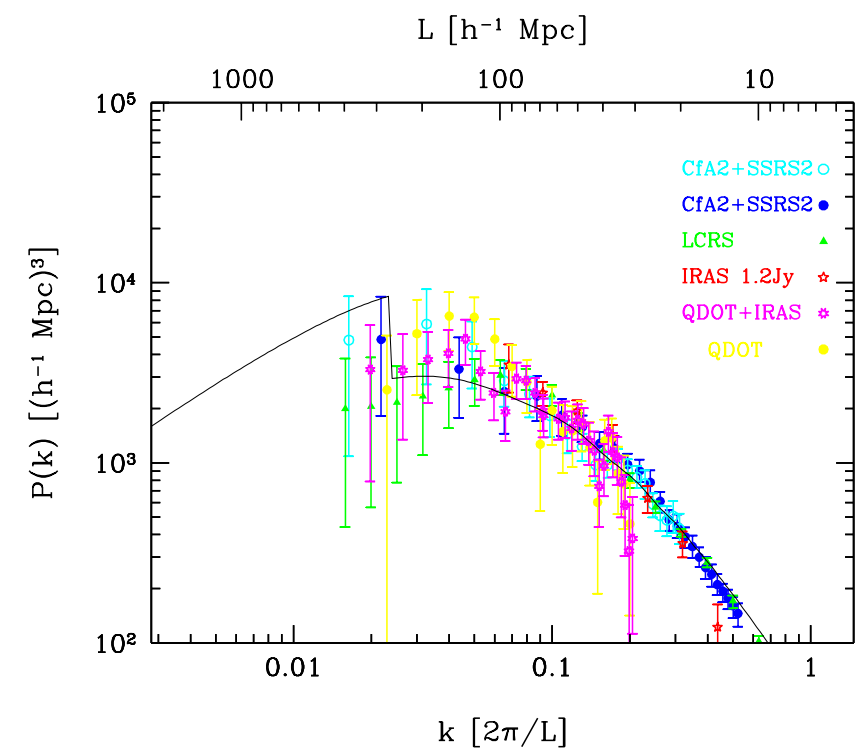

FIG. 5. The power spectrum for $k_{b}=0.023746 h^{-1} \mathrm{Mpc}, \mathcal{R}=0.345$, and $n_{\text {new }}=0.8$. $\chi^{2} /$ d.o.f $=1.07$.

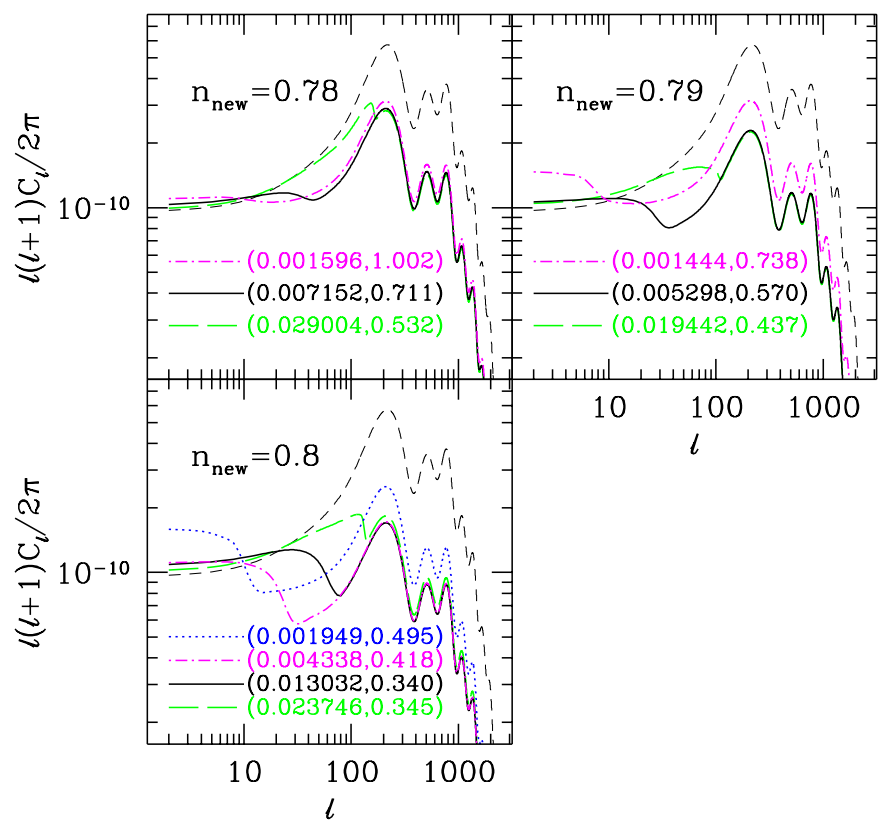

FIG. 6. The CMB angular power spectra for the nine parameter sets in Table II. $\left(k_{b}, \mathcal{R}\right)$ with $\Omega_{0}=1, h=0.5$, and $\Omega_{B}=0.06$ (baryon density parameter) are shown in the figure. In a panel for $n_{\text {new }}=0.8$, the parameter used in the previous figure is also plotted. A standard CDM model with same cosmological parameters is plotted in each panel (a dashed line) for a reference. 


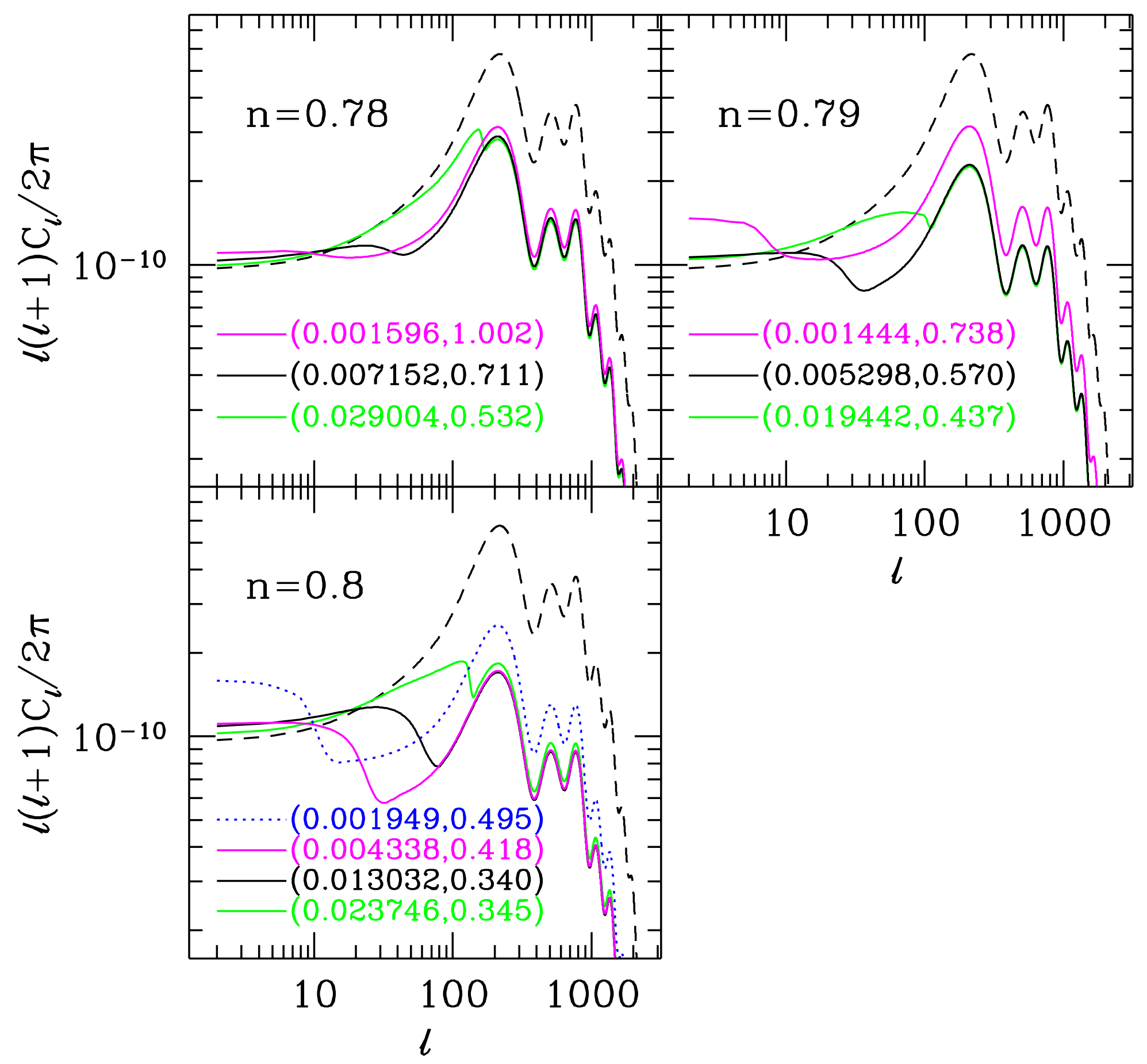

